\title{
Is Gram staining still useful in prosthetic joint infections?
}

\author{
Marjan Wouthuyzen-Bakker ${ }^{\bowtie}$, Noam Shohat ${ }^{2,3}$, Marine Sebillotte ${ }^{4}$, Cédric Arvieux ${ }^{4,5}$, Javad Parvizi², Alex \\ Soriano ${ }^{6}$ \\ 1. Department of Medical Microbiology and Infection Prevention, University of Groningen, University Medical Center Groningen, the Netherlands. \\ 2. Rothman Institute at Thomas Jefferson University Hospital, Philadelphia, United States. \\ 3. Department of Orthopaedic Surgery, Tel Aviv University, Tel Aviv, Israel. \\ 4. Department of Infectious Diseases and Intensive Care Medicine, Rennes University Hospital, Rennes, France. \\ 5. Great West Reference centers for Complex Bone and Joint Infections (CRIOGO), France. \\ 6. Service of Infectious Diseases, Hospital Clínic, University of Barcelona, Barcelona, Spain. \\ $\square$ Corresponding author: Marjan Wouthuyzen-Bakker, internist-infectiologist, University Medical Center Groningen, Department of Medical Microbiology and \\ Infection Prevention, Hanzeplein 1, 9713 GZ, Groningen, the Netherlands. Email: m.wouthuyzen-bakker@umcg.nl; Tel: + 31503616161 ; Fax: + 31503619105 \\ (c) Ivyspring International Publisher. This is an open access article distributed under the terms of the Creative Commons Attribution (CC BY-NC) license \\ (https://creativecommons.org/licenses/by-nc/4.0/). See http://ivyspring.com/terms for full terms and conditions.
}

Received: 2018.11.07; Accepted: 2018.12.19; Published: 2019.01.29

\begin{abstract}
Introduction: Staphylococcus aureus is an independent risk factor for DAIR failure in patients with a late acute prosthetic joint infection (PJI). Therefore, identifying the causative microorganism in an acute setting may help to decide if revision surgery should be chosen as a first surgical approach in patients with additional risk factors for DAIR failure. The aim of our study was to determine the sensitivity of Gram staining in late acute $S$. aureus PJI.

Material and methods: We retrospectively evaluated all consecutive patients between 2005-2015 who were diagnosed with late acute PJI due to S. aureus. Late acute PJI was defined as the development of acute symptoms and signs of PJl, at least three months after the index surgery. Symptoms existing for more than three weeks were excluded from the analysis. Gram staining was evaluated solely for synovial fluid.

Results: A total of 52 cases were included in the analysis. Gram staining was positive with Gram positive cocci in clusters in 31 cases (59.6\%). Patients with a C-reactive protein (CRP) >150 mg/L at clinical presentation had a significantly higher rate of a positive Gram stain $(30 / 39,77 \%)$ compared to patients with a CRP $\leq 150 \mathrm{mg} / \mathrm{L}(4 / 10,40 \%)(p=0.02)$. A positive $\mathrm{Gram}$ stain was not related to a higher failure rate $(60.6 \%$ versus $57.9 \%$, $p$ 0.85).

Conclusion: Gram staining may be a useful diagnostic tool in late acute PJI to identify S. aureus PJI. Whether a positive Gram stain should lead to revision surgery instead of DAIR should be determined per individual case.
\end{abstract}

Key words: Prosthetic joint infection, acute, hematogenous, Gram staining, Staphylococcus aureus

\section{Introduction}

Late acute prosthetic joint infections (PJI), which are presumed to be mostly hematogenous in origin, have a relatively high failure, especially when caused by Staphylococcus aureus [1-5]. In an acute setting, surgical debridement is often performed for immediate load reduction, but revision surgery might be the preferred treatment modality in certain patient groups. We recently demonstrated in a large cohort of late acute PJIs that patients with a preoperative
CRIME80 score (i.e. C-reactive protein $>150 \mathrm{mg} / \mathrm{L}$, Chronic obstructive pulmonary disease, Rheumatoid arthritis, fracture as Indication for the prosthesis, Male gender, not Exchanging the mobile components during debridement and an age above 80 years) above 2 , have an average failure rate of $64 \%$ and increases to $77 \%$ when $S$. aureus is involved. [1]. In addition, $S$. aureus was an independent risk factor for failure in the multivariate analysis. For this reason, identifying the 
causative micro-organism preoperatively might be helpful to choose the best primary surgical approach per individual case. In this respect, rapid microbiological detection, preferably within a couple of hours, is mandatory. So far, Gram staining has shown a poor sensitivity $(\sim 30 \%)$ in diagnosing PJI, and therefore, it clinical utility appears to be low [6-23]. However, most of the performed studies have been conducted in revision surgeries comprising mostly chronic PJIs. Considering the above mentioned clinical implications, we evaluated the sensitivity of Gram staining in a cohort of late acute PJIs caused by S. aureus.

\section{Material and Methods}

We retrospectively collected data of all consecutive patients diagnosed with a late acute $S$. aureus PJI between January 2005 and December 2015 in four University Hospitals. The studied cohort comprised a part of a larger analysis on late acute PJI treated with DAIR [1]. Late acute PJI was defined as the development of acute symptoms and signs of a PJI (i.e. a sudden onset of pain and/or swelling of the prosthetic joint) in a prior asymptomatic joint more than 3 months after the index arthroplasty. Patients with symptoms existing for longer than 3 weeks before surgery was performed were excluded from the analysis. Surgery consisted of a DAIR procedure (with or without the exchange of mobile components) or revision surgery (i.e. one- or two-stage exchange). During surgery, multiple intraoperative tissues and synovial fluid were obtained for culture and used as gold standard. As tissues samples are not available preoperatively, Gram staining performed on intraoperative biopsies were excluded from the analysis and solely analyzed for synovial fluid. In every case, one Gram staining on synovial fluid was performed. A Gram stain was considered positive for S. aureus when Gram positive cocci in clusters were observed by the medical microbiologist. S. aureus infection was confirmed with $\geq 1$ positive culture of periprosthetic intraoperative tissue or synovial fluid. PJI was defined according to the diagnostic criteria described by the Musculoskeletal Infection Society (MSIS) [24]. Multiple variables on patient characteristics, clinical presentation and outcome were collected and analyzed.

Failure was defined as: i) the need for prosthesis removal (in case revision surgery was applied as first surgical strategy, removal of the revised prosthesis was considered as failure), ii) the need for suppressive antibiotic therapy because of persistent or recurrent clinical or biochemical signs of infection, or iii) infection-related death.

Differences in patient characteristics between
Gram positive versus Gram negative cases were analyzed using a Fisher exact test with categorical variables and a Mann Whitney $U$ test with continuous variables. A p-value $<0.05$ was considered as statistical significant. Data were presented as medians \pm the interquartile range (IQR).

\section{Results}

In the studied period, a total of 66 patients were diagnosed with late acute $S$. aureus PJI. Gram staining on synovial fluid was not performed in 15 cases, leaving a total of 52 cases for analysis, comprising 2 shoulders, 18 hips and 32 knees. The majority of cases were monomicrobial infections (50 cases, 96.1\%). One of the two polymicrobial infections included a co-infection with Staphylococcus epidermidis, the other case was a co-infection with a Gram-negative rod. Gram staining was positive with Gram positive cocci in clusters in 31 cases, resulting in an overall sensitivity of 59.6\% (CI $45.1-73.0)$. Excluding missing data, patients with a C-reactive protein (CRP) $>150$ $\mathrm{mg} / \mathrm{L}$ at clinical presentation had a significantly higher rate of a positive Gram stain (30/39, 77\%) compared to patients with a CRP $\leq 150 \mathrm{mg} / \mathrm{L} \mathrm{(4/10,}$ $40 \%)(p=0.02)$. The sensitivity of Gram staining was also higher in patients with a BMI > $35(8 / 10,80 \%)$ compared to patients with a BMI $\leq 35(15 / 27,55.6 \%)$, a temperature $>38.5$ degrees Celsius $(15 / 19,78.9 \%)$ compared to a temperature $\leq 38.5$ degrees Celsius $(19 / 31,62.0 \%)$ and in patients with $S$. aureus bacteremia (22/28, 78.6\%) compared to patients with negative bloodcultures (12/22, 54.5\%). However, all these differences were not statistically different. Combining the presence of fever and a CRP > 150 $\mathrm{mg} / \mathrm{L}$, resulted in a sensitivity of $83.3 \%$ (CI 51.9 95.7). The median duration of symptoms was 3 days (IQR 1 - 7) in Gram positive cases versus 2 days (IQR 1 - 9.5) in Gram negative cases $(p=0.61)$. The sensitivity of Gram staining did not differ for the infected joint (knee or hip).

Fourty-seven patients (90.4\%) were treated with irrigation and debridement (DAIR). The remaining patients were treated with revision surgery. Overall, failure within the debrided group was $68.1 \%$ (32/47). All of the 32 failed cases, failed during antibiotic therapy. The failure rate of the revised cases was: 1 out of $5(20 \%)$. A positive Gram stain was not related with a higher failure rate compared to a negative Gram stain $(65.5 \%$ versus $72.2 \%, p=0.75)$.

\section{Discussion}

We demonstrated a moderate sensitivity (i.e. $60 \%$ ) of Gram staining in synovial fluid to detect $S$. aureus in late acute PJIs. Sensitivity was significantly higher in patients with a high CRP, probably due to a 
higher bacterial inoculum. Although the sensitivity of Gram staining was moderate, it was significantly higher in our study compared to previous reports (Table 1) [6-23]. To illustrate, a meta-analysis conducted by Ouyang et al. including a total of 4647 patients, demonstrated an overall sensitivity of Gram staining of merely $19 \%$. Although the type of infection was not always clearly defined in the included studies, it is most likely that the majority of these cases comprised chronic infections as all studies were performed in revision surgeries. This may explain the higher diagnostic yield of Gram staining in our study analyzing solely (late) acute infections.

Table 1. Studies performed on Gram staining in revision surgery. This Table was partially duplicated from the meta-analysis performed by Ouyang et al. [25].

\begin{tabular}{|c|c|c|c|c|c|c|c|c|}
\hline Author & Year & $\mathrm{N}$ & $\begin{array}{l}\mathrm{N}(\%) \text { of } \\
\text { S. aureus }\end{array}$ & Sample & Sens & Spec & PPV & NPV \\
\hline $\begin{array}{l}\text { Kraemer et } \\
\text { al. [12] }\end{array}$ & 1993 & 55 & $1(1.8)$ & Tissue & $23 \%$ & $100 \%$ & $100 \%$ & $81 \%$ \\
\hline $\begin{array}{l}\text { Feldman et } \\
\text { al. [11] }\end{array}$ & 1995 & 33 & $3(9)$ & Synovial fluid & $20 \%$ & ND & ND & ND \\
\hline $\begin{array}{l}\text { Chimento et } \\
\text { al. [10] }\end{array}$ & 1996 & 169 & No data & Tissue & $0 \%$ & $0 \%$ & $0 \%$ & $0 \%$ \\
\hline $\begin{array}{l}\text { Barrack et al. } \\
\text { [9] }\end{array}$ & 1997 & 67 & $6(8.9 \%)$ & Synovial fluid & $10 \%$ & $100 \%$ & ND & ND \\
\hline $\begin{array}{l}\text { Atkins et al. } \\
{[22]}\end{array}$ & 1998 & 297 & $3(1)$ & $\begin{array}{l}\text { Synovial fluid } \\
\text { and tissue }\end{array}$ & $6 \%$ & $100 \%$ & ND & ND \\
\hline $\begin{array}{l}\text { Della Valle et } \\
\text { al. [21] }\end{array}$ & 1999 & 413 & $14(3.4)$ & Tissue & $15 \%$ & $99 \%$ & $71 \%$ & $85 \%$ \\
\hline $\begin{array}{l}\text { Spangehl et } \\
\text { al. [20] }\end{array}$ & 1999 & 202 & No data & Tissue & $19 \%$ & $98 \%$ & $63 \%$ & $89 \%$ \\
\hline $\begin{array}{l}\text { Virolainen et } \\
\text { al. [18] }\end{array}$ & 2002 & 68 & $15(22)$ & Synovial fluid & $67 \%$ & $100 \%$ & ND & ND \\
\hline $\begin{array}{l}\text { Banit et al. } \\
{[19]}\end{array}$ & 2002 & 121 & $10(8 \%)$ & Tissue swab & $43 \%$ & $100 \%$ & ND & ND \\
\hline Ko et al. [17] & 2005 & 40 & 7 (17.5) & Tissue & $0 \%$ & $0 \%$ & $0 \%$ & $0 \%$ \\
\hline $\begin{array}{l}\text { Trampuz et } \\
\text { al. [23] }\end{array}$ & 2007 & 326 & $10(12.6)$ & $\begin{array}{l}\text { Sonication } \\
\text { fluid }\end{array}$ & $45 \%$ & $100 \%$ & $100 \%$ & $86 \%$ \\
\hline $\begin{array}{l}\text { Parvizi et } \\
\text { al.[8] }\end{array}$ & 2006 & 70 & $26(27.6)$ & Synovial fluid & $35 \%$ & $97 \%$ & $94 \%$ & $54 \%$ \\
\hline $\begin{array}{l}\text { Ghanem et al. } \\
\text { [7] }\end{array}$ & 2009 & 1004 & No data & Tissue & $31 \%$ & $100 \%$ & $99 \%$ & $75 \%$ \\
\hline $\begin{array}{l}\text { Morgan et al. } \\
\text { [6] }\end{array}$ & 2009 & 921 & No data & $\begin{array}{l}\text { Synovial fluid } \\
\text { and tissue }\end{array}$ & $27 \%$ & $100 \%$ & $99 \%$ & $79 \%$ \\
\hline $\begin{array}{l}\text { Johnson et al. } \\
\text { [16] }\end{array}$ & 2010 & 202 & No data & Tissue swab & $10 \%$ & $100 \%$ & $100 \%$ & $62 \%$ \\
\hline $\begin{array}{l}\text { Oethinger et } \\
\text { al. [15] }\end{array}$ & 2011 & 269 & No data & $\begin{array}{l}\text { Synovial fluid } \\
\text { and tissue }\end{array}$ & $9 \%$ & $99 \%$ & ND & ND \\
\hline $\begin{array}{l}\text { Schindler et } \\
\text { al. [14] }\end{array}$ & 2011 & 62 & $15(24.2)$ & NS & $2 \%$ & ND & ND & ND \\
\hline $\begin{array}{l}\text { Zywiel et al. } \\
\text { [13] }\end{array}$ & 2011 & 347 & No data & Tissue swab & $7 \%$ & $99 \%$ & $92 \%$ & $57 \%$ \\
\hline
\end{tabular}

Sens: sensitivity, Spec: specificity, PPV: positive predictive value, NPV: negative predictive value, ND: not determined.

As late acute $S$. aureus PJI treated with DAIR is associated with a relatively high failure rate, especially in patients with a high preoperative CRIME80 score [1], a Gram stain with Gram positive cocci in clusters may be useful in an acute setting to consider revision surgery instead of DAIR. Previous studies have shown that late acute PJI caused by microorganisms other than $S$. aureus, have a better outcome, and a DAIR procedure may be feasible in the majority of these patients [1, 3-4]. Therefore, especially when immediate load reduction is warranted, rapid preoperative identification of the causative microorganism might be helpful to determine the primary surgical approach. Until now, molecular detection does not show any benefit in acute PJIs [26]. Although a negative Gram stain does not exclude an infection caused by $S$. aureus as indicated by our results, the specificity and positive predictive value of Gram stain appears to be very high as previously demonstrated [25.] Therefore, a high CRIME80 score combined with a Gram stain containing Gram positive cocci in clusters may guide the orthopaedic surgeon to consider revision surgery instead of DAIR. Although the presence of coagulase negative staphylococci (CoNS) cannot be excluded in Gram stains with positive cocci in clusters, these microorganisms are very rare in causing late acute infections [1]. Moreover, if CoNS are isolated, the chance that the PJI is a previously unrecognized chronic PJI, instead of a late acute PJI, is much more likely. In these cases, a revision surgery instead of a DAIR would be the preferred treatment modality as well [27]. Obviously, the ultimate decision to perform revision surgery should be made per individual case and preferably discussed within a multidisciplinary team as several factors needs to be taken into account.

In conclusion, our results indicate that implementing Gram staining in routine diagnostics may have additional clinical value in late acute PJIs as the presence of Gram positive cocci in cluster may warrant the surgeon to intensify surgical treatment in certain patient groups.

\section{Competing Interests}

The authors have declared that no competing interest exists.

\section{References}

1. Wouthuyzen-Bakker M, Sebillotte M, Lomas J et al. Clinical outcome and risk factors for failure in late acute prosthetic joint infections treated with debridement and implant retention. Journal of Infection 2018; in press.

2. Lora-Tamayo J, Murillo O, Iribarren JA et al. A large multicenter study of methicillin susceptible and methicillin-resistant Staphylococcus aureus prosthetic joint infections managed with implant retention. Clin Infect Dis 2013; 56(2):182-94.

3. Vilchez F, Martinez-Pastor JC, Garcia-Ramiro S et al. Efficacy of debridement in hematogenous and early post-surgical prosthetic joint infections. Int J Artif Organs 2011; 34(9):863-9.

4. Rodriguez D, Pigrau C, Euba G et al. Acute haematogenous prosthetic joint infection: prospective evaluation of medical and surgical management. Clin Microbiol Infect 2010; 16(12):1789-95.

5. Tande AJ, Palrai BR, Osmon DR et al. Clinical presentation, risk factors, and outcomes of hematogenous prosthetic joint infection in patients with Staphylococcus aureus bacteremia. Am J Med 2016; 129(2):e11-20.

6. Morgan PM, Sharkey P, Ghanem E et al. The value of intraoperative Gram stain in Revision total knee Arthroplasty. J Bone Joint Surg Am 2009; 91:2124-9.

7. Ghanem E, Ketonis C, Restrepo C et al. Periprosthetic infection: where do we stand with regard to Gram stain? Acta Orthopaedica 2009; 80(1):37-40.

8. Parvizi J, Ghanem E, Menashe S, Barrack RL and Bauer TW: Periprosthetic infection: what are the diagnostic challenges? J Bone Joint Surg Am 88 Suppl 4: 138-147, 2006. 
9. Barrack RL, Jennings RW, Wolfe MW and Bertot AJ: The Coventry Award. The value of preoperative aspiration before total knee revision. Clin Orthop Relat Res: 8-16, 1997.

10. Chimento GF, Finger S and Barrack RL: Gram stain detection of infection during revision arthroplasty. J Bone Joint Surg Br 78: 838-839, 1996.

11. Feldman DS, Lonner JH, Desai P and Zuckerman JD: The role of intraoperative frozen sections in revision total joint arthroplasty. J Bone Joint Surg Am 77: 1807-1813, 1995.

12. Kraemer WJ, Saplys R, Waddell JP and Morton J: Bone scan, gallium scan and hip aspiration in the diagnosis of infected total hip arthroplasty. J Arthroplasty 8: 611-616, 1993

13. Zywiel MG, Stroh DA, Johnson AJ, Marker DR and Mont MA: Gram stains have limited application in the diagnosis of infected total knee arthroplasty. Int J Infect Dis 15: e702-e705, 2011.

14. Schindler M, Christofilopoulos P, Wyssa B, et al: Poor performance of microbiological sampling in the prediction of recurrent arthroplasty infection. Int Orthop 35: 647-654, 2011.

15. Oethinger $M$, Warner DK, Schindler SA, Kobayashi $H$ and Bauer TW: Diagnosing periprosthetic infection: false-positive intraoperative Gram stains. Clin Orthop Relat Res 469: 954-960, 2011.

16. Johnson AJ, Zywiel MG, Stroh DA, Marker DR and Mont MA: Should gram stains have a role in diagnosing hip arthroplasty infections? Clin Orthop Relat Res 468: 2387-2391, 2010.

17. Ko PS, Ip D, Chow KP, Cheung F, Lee OB and Lam JJ: The role of intraoperative frozen section in decision making in revision hip and knee arthroplasties in a local community hospital. J Arthroplasty 20: 189-195, 2005.

18. Virolainen $\mathrm{P}$, Lähteenmäki $\mathrm{H}$, Hiltunen A, Sipola E, Meurman $\mathrm{O}$ and Nelimarkka O: The reliability of diagnosis of infection during revision arthroplasties. Scand J Surg 91: 178-181, 2002.

19. Banit DM, Kaufer $\mathrm{H}$ and Hartford JM: Intraoperative frozen section analysis in revision total joint arthroplasty. Clin Orthop Relat Res: 230-238, 2002.

20. Spangehl MJ, Masterson E, Masri BA, O'Connell JX and Duncan CP: The role of intraoperative gram stain in the diagnosis of infection during revision total hip arthroplasty. J Arthroplasty 14: 952-956, 1999.

21. Della Valle CJ, Scher DM, Kim YH, et al: The role of intraoperative Gram stain in revision total joint arthroplasty. J Arthroplasty 14: 500-504, 1999.

22. Atkins BL, Athanasou N, Deeks JJ, et al: Prospective evaluation of criteria for microbiological diagnosis of prosthetic-joint infection at revision arthroplasty. The OSIRIS Collaborative Study Group. J Clin Microbiol 36: 2932-2939, 1998.

23. Trampuz A, Piper KE, Jacobson MJ et al. Sonication of removed hip and knee prostheses for diagnosis of infection. N Engl J Med 2007; 357(7): 654-63.

24. Parvizi J and Gehrke T. Definition of periprosthetic joint infection. J Arthroplasty 2014; 29(7):1331

25. Ouyang Z, Zhai Z, Qin AN et al. Limitations of Gram staining for the diagnosis of infections following total hip or knee arthroplasty. Meta-analysis. Exp Ther Med 2015; 9(5): 1857-64.

26. Villa F, Toscano M, De Vecchi E et al. Reliability of a multiplex PCR system for diagnosis of early and late prosthetic joint infections before and after broth enrichment. Int J Med Microbiol 2017; 307(6):363-70.

27. Barberán J, Aguilar L, Carroquino G, Giménez MJ, Sánchez B, Martínez D, Prieto J. Conservative treatment of staphylococcal prosthetic joint infections in elderly patients. Am J Med 2006; 119(11):993.e7-10. 